\title{
No Application Type
}

National Cancer Institute

\section{Source}

National Cancer Institute. No Application Type. NCI Thesaurus. Code C123271.

There is no associated application type (e.g., as with an over-the-counter monograph drug), and therefore this field is not applicable. 